\title{
Urologic Management of the Spinal Cord Injured Patient
}

\author{
Reynaldo G. Gómez ${ }^{1}$. Sean P. Elliott ${ }^{2}$
}

Published online: 10 September 2018

c) Springer-Verlag GmbH Germany, part of Springer Nature 2018

In this issue of the World Journal of Urology we present a condensed summary of the 2016 joint Consultation of the Société Internationale d'Urologie (SIU) and the International Consultation for Urologic Disease (ICUD) on the Urologic Management of the Spinal Cord Injured Patient (SCI).

This is a complex, poorly understood and often-neglected topic. It is challenging because it compromises not only the physical sphere of the patient, but also severely affects the affective, social, economic and occupational sphere, presenting a formidable challenge of rehabilitation.

A few decades ago, urologic complications, mainly renal failure and urosepsis, accounted for the majority of long-term deaths after SCI. The advent of urodynamics and intermittent catheterization and significant advances in both medical and surgical therapy has dramatically altered the course of disease. However, despite these advances, there is a widespread lack of knowledge around the world about the best management of these patients, and the quality of care is deficient even in many developed countries. We all encounter these patients in our practice, yet we lack a central repository for the body of information on this topic. This SIU-ICUD consultation seeks to fill that gap.

This consultation was performed by a panel of about 80 experts, which included not only urologists, but also neurologists, physiatrists and therapists, with a global representation of continents, cultures and socioeconomic status to have a global perspective.

Since this is a very wide and heterogeneous topic, it was originally divided into nine chapters dealing not only with the management of the urinary problems, but also with the

Reynaldo G. Gómez

gomez.reynaldo@gmail.com

Sean P. Elliott

selliott@umn.edu

1 Universidad Andres Bello School of Medicine and Urology Service Hospital del Trabajador, Ramon Carnicer 185, Santiago, Chile

2 University of Minnesota, 420 Delaware Street SE; MMC 394, Minneapolis, MN 55455, USA management of the bowel, fertility and sexuality. A separate consideration was given to the management of children and elderly patients, and in the last chapter a global perspective of the current patterns of care and future directions was presented.

A committee was appointed for each of the nine chapters and each chapter committee performed a comprehensive review of the pertinent literature for their respective chapters. Conclusions and recommendations were then produced based on the published evidence and organized according to the ICUD modification of the Oxford grading system for evidence-based medicine (Tables 1 and 2).

In October 2016, each of the chapter chairs presented a summary of their work during the 36th Congress of the Société Internationale d'Urologie in Buenos Aires, Argentina. The discussion that ensued from those presentations was also incorporated into the final book of the consultation, which was released at the 2017 SIU meeting in Lisbon.

The contents of these nine chapters have been condensed to fulfill the publication guidelines of the journal and, because of their original large size, two of the chapters were split into two, generating the 11 sections of this issue.

This consultation summarizes what it is available at this time. However, despite the valuable information already available, high-quality studies are still insufficient and there are numerous areas in great need for research, so this work may also help to direct that research.

We want to express our gratitude to the World Journal of Urology for the opportunity to publish this material and for entrusting us with the editorial task of this important project. We are sure that this topic issue will help to better disseminate this updated knowledge for the benefit of the patients around the world. 
Table 1 Summary of the International Consultation On Urological Disease Modified Oxford Centre for Evidence-Based Medicine Grading System for Guideline Recommendations

Table 2 Summary of the International Consultation on Urological Disease Modified Oxford Centre for Evidence-Based Medicine Grading System for Guideline Recommendations

\begin{tabular}{ll}
\hline Levels of evidence & Description \\
\hline I & Meta-analysis of RCTs or high-quality RCT \\
II & Low-quality RCT or good-quality prospective cohort study \\
III & Good quality retrospective case-control study or cohort \\
& study \\
IV & Expert opinion \\
\hline
\end{tabular}

$R C T$ randomized controlled trail

\section{Grades of recommendation Description}

\begin{tabular}{ll} 
A & Usually consistent with level I evidence \\
B & Consistent level II or III evidence or "majority evidence" from RCTs \\
C & Level IV evidence or "majority evidence" from level II or III studies \\
D & No recommendation possible because of inadequate or conflicting evidence \\
\hline
\end{tabular}

$R C T$ randomized controlled trail 